\title{
A Safe Space to Speak Above the Silences
}

\author{
Roxana Llerena-Quinn
}

Published online: 14 May 2013

(C) Springer Science+Business Media New York 2013

A few years ago two social scientists attended a faculty development course on cultural awareness that my colleagues and I offered to medical school faculty. Their research, reported in their paper in this collection, "lifts the hood" on the course, as we tried to do with each other in order to allow us, the faculty, to see ourselves and the course through the eyes of others. Their inclusionary approach of engaging all the voices offers an additional feedback loop now as they invite me to share some comments on their paper and on the course. The process of producing this essay evokes many possibilities; not only for the course, but also regarding the inherent value of making space for voice and reflection in the construction of knowledge. Unavoidably, my comments on the previous article are colored by the perspective of a behavioral health clinician, just one lens of many possible others in the broader discussion of health.

Currently, in the United States, we are at a crossroads as a result of health care reform (the Affordable Care Act (ACA)) and budget pressures. Racial/ethnic health and health care disparities in the U.S. are well-documented (Smedley et al. 2003). During the past decade, mental health care disparities have persisted for black and Latino youth in a 2-to-1, white-to-minority ratio despite efforts to reduce them, and the gap is growing even wider for Latino children (Cook et al. 2013). This is alarming given that children's mental illness is predictive of a variety of poor outcomes later in life. Social advocates need to follow these changes carefully and come together in force to insure that the health care system that emerges as a result of current reforms is more responsive to people of all cultures and social classes. I lose hope for this kind of change when I hear comments from well-intentioned colleagues who make statements like "we are more concerned about the $75 \%$ percent of children," or who state that public insurance patients are money losers

R. Llerena-Quinn ( $\bowtie)$

Boston Children's Hospital, Harvard Medical School, 300 Longwood Ave., Fegan 8,

Boston, MA 02115, USA

e-mail: roxana.1lerena-quinn@childrens.harvard.edu 
and that "we cannot be all things to all people." In the meantime, for the first time in history, more minority children (50.4 births) than white children were born in the U.S. (U.S. Census Bureau 2011). More than ever before, the well-being of our nation depends on the well-being of all the children. My hope is that when these children grow up they will see their peer cohorts as part of the same nation.

\section{A Corner of Silence Among Other Silences}

I was motivated to develop and teach courses on cultural awareness because of my own experiences as a Latina care provider in a 'monocultural' clinical culture. Like many of my contemporaries, I was trained in a monocultural curriculum concerned with human suffering but in which the poor and minorities were seen as untreatable. Unexamined assumptions about why the poor or minorities "fail to show improvement and/or leave treatment" served to justify their exclusion while leaving unanswered the question of why mainstream mental health services might be unacceptable to these populations. Unlike the formal curriculum, the informal curriculum in my training practice provided a safe space to voice and challenge existing epistemologies that prevented us from seeing that Latino children "belong to everyone" or that we must work together to address the gaps. This informal curriculum provided a framework to understand the role of structural and social forces in producing social suffering and the varying degrees of personal control in people's lives, to change them. Today, we are formally taught to value diversity, while our practices through standardization are becoming increasingly monocultural.

As mental health disparities continue to worsen for Latinos despite recognition that "culture matters" (USDHHS 2001), we are then called to attend to the hidden curriculum $^{1}$ that lies beneath institutional claims of cultural competence and valuing diversity. This hidden curriculum is revealed in some training programs when, for example, minority interns are asked to teach the seminar session on cultural competence regardless of preference or interest. Curiously, it has been my experience that when some Latino clinicians apply their cultural knowledge into practice, they are challenged by some non-Latino supervisors who question their therapeutic boundaries in the process of engaging with Latino families. For example, a supervisor may find it a boundary violation to accept a cup of coffee during an in-home therapy visit, whereas the clinician may understand that accepting this simple act of hospitality is crucial to establishing rapport. The message remains that "culture" is still something "others" have, "cultural knowledge" is not essential to clinical excellence (cf. Bullon this volume), and providers who have this kind of knowledge are not seen as competent. Meanwhile, the culture of medicine and the culture of mainstream providers remain unmarked and continue to define the "right and proper" way to do things.

\footnotetext{
${ }^{1}$ The concepts of the informal and hidden curriculum emphasize the latent, implicit learning that occurs outside of formal classroom settings in medical education. These concepts call attention to the importance of informal interpersonal interactions and structural factors (Hafferty 1998).
} 
It behooves us to understand why such things are happening at a time when the Surgeon's General's 2001 Report on Mental Health: Culture Matters and the 2003 IOM Report on Unequal Treatment officially claim the importance of "culture" in biomedicine and mental health and when "cultural competence" courses and trainings are appearing at all levels of medical and mental health education.

\section{Pedagogy: "Cultural Awareness" Versus "Cultural Competence"}

The faculty course discussed by the preceding paper evolved from a course originally offered to medical students through the Department of Social Medicine. The student course itself developed in response to demands by an increasingly diverse student body for a curriculum that would prepare them to practice medicine in the communities from which they came. While it is understood that health and health inequities arise from complex multiple factors within and outside the practice of medicine, initial school efforts to integrate multicultural education into the curriculum raised concerns about stereotyping. Concerns about stereotyping in the overt and the hidden curriculum were difficult to talk about openly. To address the silences, a demographically and professionally diverse group of faculty, capitalizing on their own diversity for mutual learning, developed a course where students, like them, could explore and reflect on own their own cultural identities with each other and learn about the inherent dynamics that emerge when differences interact. Unlike old traditional notions of cultural competence as the detached mastery of a finite body of knowledge about the other, our approach to cultural competence and of encouraging self-awareness aligns with the notion of "cultural humility." 2 The course never intended to become the only response for understanding the complex multilayered responses that are needed to provide equitable care or address social inequities. Rather, the pedagogy of self-awareness is seen as a necessary, but insufficient first step in the lifelong journey toward understanding and developing accountable personal and institutional relationships with similar and different others.

Success with this kind of pedagogy depends on the instructors' ability to create a safe "holding environment" in which differences can be voiced, heard, and engaged with respect. The "no blame" interactional environment encouraged in the course does not turn a blind eye to the social structures and practices that produce people harm. Rather, its goal is to reduce the intergroup anxiety that produces aversive racism and prevents mutual learning. This process does not imply the absence of strong emotions; in fact, these emotions were often cited in student journals as catalysts for learning. The pedagogy of "self-awareness" focuses on the self and does not seek to become an "expert" about the other. The focus on the self is useful as long as one takes ownership of one's own history and life biases and, moreover,

\footnotetext{
${ }^{2}$ The concept of cultural humility incorporates a life-long commitment to self-evaluation and reflexive self-critique, to redressing the power imbalances and to developing mutually beneficial partnerships with communities on behalf of individuals and defined populations (Tervalon and Murray-García 1998).
} 
as long as the "expertise" attributed to oneself or others is tempered by humility regarding how much remains to be learned from one another.

The faculty course evolved from students' suggestions that the course should be offered to faculty, who were believed to be less diverse than the student body and who might benefit from exploring these issues. The faculty course was sponsored by another department at the school whose mission centers on faculty development on teaching.

Both courses were elective, which insured high motivation and were taught by the same faculty with few changes in the current iteration. The faculty course was in its third iteration, the student course in its eighth. The student course was offered to medical students at the beginning of their medical careers, while participants in faculty course were well established in their careers.

\section{Reflections on the Faculty Course}

The faculty course examined in the preceding article had a new title from previous iterations, it focused on "bias", and introduced new topics: "teaching tips" (expert stance) and case discussion (focus on the other). The changes reflected the sponsoring department's mission and emerging questions among experts as to the value of selfawareness versus a more skill-based, case-approach. Though both approaches are important to build on the other, the need to separate them is more obvious now.

First, the instructors were distracted by new unfamiliar tasks that took attention away from process; they were more experienced with previous iterations of the faculty course - "Self-Awareness and Cultural Identity in Medicine and Beyond"that focused only on self-awareness. Second, the new tasks re-directed attention to the "other" rather than on the self. Third, it misled those who wanted a more cognitive, skills-based course instead of introspection. Last, there was less time to focus on the single most important goal of the course, which was to increase the degree of comfort in talking about and engaging difference to prevent aversive withdrawal.

Since success with this kind of pedagogy depends on the instructors' ability to create a safe "holding environment" some possible factors contributing to a breach in this area are highlighted here:

1. Time pressure to cover less familiar topics in less time created an unintended hidden curriculum of conflict avoidance reinforced by the message: "we can't talk about this any further, and we need to move on". People may not be likely to open up an important topic (particularly a deeply emotional one) if they know they are likely to be interrupted or cut short. Unfortunately, this leads to superficial exploration of important themes that emerge. For example, the role that institutional cultures and the culture of medicine have on quality of care as they tempt providers to exclude patients who are "others", who take more time or are not "efficient" in their own care. Not unlike clinical practice, time pressure in the classroom prevented the exploration of richer narratives that would defy monolithic or stereotypical notions of culture. 
2. The experience of difference in the classroom or other settings can be silencing if the environment is not perceived as a safe one in which to speak. Failure to engage the minority voice, with curiosity and from a not-knowing position, can prevent everyone from learning from that difference by allowing it to withdraw into silence untouched. For example, the post-course interviews (as detailed in the preceding paper) revealed our failure to make space for class bias attitudes to emerge and therefore, to engage in a deeper discussion on class privilege and the degree of personal control over life choices this affords. Had it been safe to voice that bias, we could have explored other forms of otherness, e.g., different forms of family structures, poverty, single parenting, immigration, parenting practices in un-affirming, or dangerous environments. We could have explored differences in how particular diagnoses are understood and attributed to different causes across social classes. Most important perhaps, there was a missed opportunity to connect with the helplessness providers may feel in the face of overwhelming patient problems that are seen as "individual" problems. A more contextual understanding could free the provider and the families themselves to explore more empowering responses.

I wish we had been able to access the faculty feedback sooner. Valuable information was missed not seeking this feedback in real time. A few ideas come to mind for the next time we teach this course:

1. Anticipate "otherness", discuss the value of this voice for learning and if it was "ours", what we would need to voice it. Emphasize the importance of staying in the conversation and not withdrawing into silence.

2. Improve structure and safeguards to process. Find ways to gain regular feedback on process and the hidden curriculum, either by checking-in or through a rotating "reflecting team" among class members.

3. Maximize ways to learn from others. Strengthen process continuity by allowing people to stay in the same small group. Written summaries of small group insights could be compiled and shared with the whole class to keep everyone on the same page and have better large group discussion.

\section{A Final Word on Provider Bias}

When clinical supervisors lack insight or a broader contextual understanding, for example, when they offer misplaced recommendations like not accepting a cup of coffee, serious ethical issues arise regarding supervision of clinical training. Still, provider bias is not the only source of bias influencing mental health disparities. Such a reductionist suggestion would deny the multidimensionality of bias and its embeddedness at all levels of the health care system.

Current changes and pressures within medical culture work against efforts to increase cultural awareness including, for example, standardization of care, econometric demands to increase productivity, and increased paperwork and time pressure (cf. Bullon this volume). As currently organized, the U.S. health system 
tells us to pay attention to diversity (variation among patients) while mental health culture has shifted towards biology, standardized treatments (that permit little if any variation), and the replacement of patient narratives with checklists (Bullon et al. 2011). Indeed, the "voice of the lifeworld" (Mishler 1984) is increasingly silenced or sidelined. Under pressure, providers are becoming increasingly more distanced from their patients, which many fear is "deleting" the relational aspects of care. These pressures put not only patients, but also providers themselves at risk.

We are pressed for time while caring for hyper-diverse patients who come from increasingly diverse family structures. They want more time with their doctors, to be seen as whole people beyond just their symptoms, and to communicate their most important concerns in a multiplicity of languages. Given these pressures, we need to pause to examine how we are advancing provisions of the ACA for family-centered care that is delivered in culturally competent organizations (Andrulis et al. 2010). Many continue to hold the hope that reform will narrow health inequities.

\section{Concluding Remarks}

In closing, I offer the following points as implications of our work in trying to teach cultural awareness:

1. An increased capacity to see the "different other" beyond stereotypes strengthens trust and relationship which are at the core of best practices. Like all of us, patients are experts about their lived experience, their experience of the illness and of us in the medical system. Treat patients as story-tellers, teachers. Be open to surprise, expect to learn something new.

2. Standardized treatments are a-contextual. It is important to understand what happens to each of us when we take away context. Our vision must expand to include the economic, political, environmental, and sociocultural determinants of health while asking what is of most concern to our patients. Contextual awareness helps see the person more clearly beyond diagnosis.

3. It is essential to remember "otherness" whether in the clinic or in the classroom. We may not see it, but it is there noticing us. Recognizing what we can gain from learning about difference, provide a safe space for voice to speak above the silences.

\section{References}

Andrulis Dennis P, Siddiqui Nadia J, Purtle Jonathan P, Duchon Lisa

2010 Patient Protection and Affordable Care Act of 2010: Advancing health equity for racially and ethnically diverse populations. Washington, DC: Joint Center for Political and Economic Studies. Uploaded April 14, 2013, http://www.jointcenter.org/hpi/sites/all/files/PatientProtection_ PREP_0.pdf.

Bullon, Antonio, Mary-Jo DelVecchio Good, and Elizabeth Carpenter-Song

2011 The Paper Life of Minority and Low-Income Patient Care. In Shattering Culture: American Medicine Responds to Cultural Diversity. M. Good, S. Willen, S. Hannah, K. Vickery, and L. Park, eds., pp. 200-216. New York: Russell Sage Foundation. 
Cook Benjamin, L, L Barry Colleen, and H. Busch Susan

2013 Racial/ethnic disparity trends in children's mental health care access and expenditures from 2002 to 2007. Health Services Research 48(1): 129-149.

Hafferty, Frederic.W.

1998 Beyond Curriculum Reform: Confronting Medicine's Hidden Curriculum. Academic Medicine 73: 403-407.

Mishler, Elliot

1984 The Discourse of Medicine: The Dialectics of Medical Interviews. Norwood, NJ: Ablex.

Smedley, B.E., A.Y. Stith, and A.R. Nelson

2003 Unequal Treatment: Confronting Ethnic and Racial Disparities in Health Care. Washington, DC: National Academies Press.

Tervalon, Melanie, and Jann Murray-García

1998 Cultural Humility Versus Cultural Competence: A Critical Distinction in Defining Physician Training Outcomes in Multicultural Education. Journal of Health Care for the Poor and Underserved 9(2): 117-125.

U.S. Department of Health and Human Services

2001 Mental Health: Culture, Race, Ethnicity—Supplement to Mental Health: Report of the Surgeon General. Rockville, MD: Government Printing Office. 